\title{
Parole publique des femmes et conflictualité pendant la Révolution, dans le Sud-Est de la france
}

\section{Martine Lapied}

\section{(2) OpenEdition \\ 1 Journals}

\section{Édition électronique}

URL : https://journals.openedition.org/ahrf/5973

DOI : 10.4000/ahrf.5973

ISSN : 1952-403X

Éditeur :

Armand Colin, Société des études robespierristes

\section{Édition imprimée}

Date de publication : 1 juin 2006

Pagination : 47-62

ISSN : 0003-4436

\section{Référence électronique}

Martine Lapied, «Parole publique des femmes et conflictualité pendant la Révolution, dans le Sud-Est de la france », Annales historiques de la Révolution française [En ligne], 344 | avril-juin 2006, mis en ligne le 01 juin 2009, consulté le 22 avril 2022. URL : http://journals.openedition.org/ahrf/5973 ; DOI :

https://doi.org/10.4000/ahrf.5973

Ce document a été généré automatiquement le 22 avril 2022.

Tous droits réservés 


\title{
Parole publique des femmes et conflictualité pendant la Révolution, dans le Sud-Est de la france
}

\author{
Martine Lapied
}

1 Sous l'Ancien Régime, les provinciales sont peu visibles dans l'espace politique. On sait que des femmes participaient parfois aux assemblées des communautés, mais leur parole est rarement rapportée dans les textes. De plus, dans le Sud-Est, une évolution oligarchique de la vie municipale au XVIII ${ }^{\mathrm{e}}$ siècle transforme ces assemblées en conseils clos ce qui rend leur présence encore plus improbable.

2 La parole des femmes devient plus facilement audible, et éventuellement visible dans les archives, en situation de crise, de conflictualité, dans les périodes d'affaiblissement de normes qui voudraient les cantonner à l'espace privé. Des émeutes aux grandes crises politiques, telles la Fronde, les révolutions, dans tous les moments de rupture, des femmes s'expriment par des actes, ou des paroles, investissant ainsi la sphère publique. Elles peuvent alors exprimer leurs points de vue et tenter de contrôler les autorités en énonçant leurs volontés ${ }^{1}$.

3 La Révolution française est marquée par l'action des femmes, elle apporte aussi des témoignages de leurs paroles, dès la rédaction des doléances. Bien qu'il existe relativement peu de traces de la parole publique des provinciales, l'exploration approfondie des fonds d'archives montre qu'elle a existé. Il s'agit de rechercher comment les femmes pouvaient faire connaître leur point de vue dans la sphère publique; recherche difficile en ce qui concerne des femmes ordinaires qui n'appartiennent pas à l'élite, qui sont souvent restées des inconnues, des anonymes dont la parole même prononcée n'est pas jugée digne d'être conservée. Cette parole a $\mathrm{du}$ mal à s'exprimer à travers les institutions, les instances officielles dont les femmes sont souvent exclues, par contre elle se fait entendre de façon privilégiée dans certains lieux de sociabilité lorsqu'il s'agit de faire œuvre de propagande pour un camp ou l'autre, mais elle n'est pas toujours rapportée. 
4 Lieu de forte conflictualité pendant la Révolution, le Sud-Est est favorable à cette expression. À partir de corpus de sources essentiellement issues des papiers des sociétés populaires, comités de surveillance et tribunaux révolutionnaires provençaux, ainsi que des journaux, l'étude essaiera de mettre en visibilité la parole publique des femmes et de montrer quelles voies elle emploie pour être entendue.

La participation aux doléances

5 Des femmes ont probablement participé, en particulier dans les campagnes, à la rédaction des doléances des communautés, mais ces apports ne peuvent être identifiés.

6 Un cahier de doléances de corporation féminine a été retrouvé, il s'agit de celui des blanchisseuses et lavandières de Marseille ${ }^{2}$. Ces femmes du peuple ne s'adressent pas au roi en tant que femmes, mais se présentent comme la catégorie la moins fortunée des citoyens. Les petits métiers féminins urbains rapportant encore moins que les métiers exercés par des hommes dans le monde de l'artisanat, les blanchisseuses et lavandières ont effectivement des conditions de vie particulièrement difficiles. Ne sachant pas écrire, elles indiquent qu'elles sont obligées d'avoir recours à un citoyen charitable pour écrire leurs plaintes. Elles affirment néanmoins leur soutien au roi dont elles attendent "les plus grands adoucissements " à leur misère et veulent montrer que malgré leur manque d'instruction, elles doivent avoir leur place dans l'espace public:

"Si nous n'avons pas la capacité de manifester nos sentiments de reconnaissance par des écrits, nous avons celle de les exprimer par des acclamations, qui quoique tumultueuses, ont plus d'énergie que tous les beaux discours qui ne frappent point au cœur: nos réjouissances exaltent mieux notre roi que toutes les belles phrases qu'on lui adresse. »

7 Les revendications, comme c'est souvent le cas dans les cahiers de corporations, sont avant tout économiques et sociales. Elles se plaignent d'être à la merci des marchands de savon qu'elles accusent de faire fortune en les exploitant. C'est davantage une conscience de classe qui se dessine ici qu'une analyse féministe de la précarité de leur situation.

Les femmes et la sociabilité révolutionnaire

8 La volonté des femmes de s'engager dans les pratiques politiques nouvelles s'affirme dans leur participation aux sociétés populaires. De façon générale, les renseignements fournis par les papiers des sociétés populaires sont parmi les témoignages les plus significatifs des mentalités et des attitudes politiques des communautés concernées. La région étudiée possède un réseau extrêmement dense de clubs.

9 Vaucluse : 134 sociétés recensées ( $92,4 \%$ des communes)

Bouches- du- Rhône : 88 (83,8\%)

Var : $136(60,4 \%)$

Basses Alpes : $147(56,5 \%)^{3}$

10 Mais nous sommes loin de disposer de documents significatifs pour l'ensemble des sociétés recensées et, en particulier, nous ignorons pour bon nombre d'entre elles si des femmes assistaient à leurs séances.

11 Pour participer au mouvement associatif, les militantes ont deux possibilités : se faire admettre dans des clubs mixtes ou créer des clubs féminins. 56 clubs de femmes ont été recensés en France entre 1789 et $1793^{4}$.

Les clubs féminins 
12 À Marseille, dans les débuts de la Révolution, "dames citoyennes et dames hérö̈nes » peuvent s'exprimer dans l'espace public, certaines de leurs proclamations sont publiées $^{5}$. Utilisant la rhétorique révolutionnaire, elles parlent un langage de la citoyenneté basé sur les droits et les devoirs qui sont issus de leur attachement aux nouvelles lois de la Nation.

13 Les dames de la Section 7, du district de Saint-Martin, s'étaient réunies en société des Dames citoyennes. Elles s'assemblaient dans la chapelle des pénitents bleus de SaintMartin. Le 7 juillet 1790, les Dames s'assemblent et élisent, par acclamation, Françoise Raimbaud présidente et Marie-Jeanne Boude secrétaire. Le procès-verbal comporte 107 signatures, celle de Madame Martin, l'épouse du maire en tête ; la plupart des délibérations issues de cette société rassemblent plus de 100 et jusqu'à 170 signatures.

Les Dames hérönes de la Section 11 avaient aussi formé leur société. Au nombre de 66, elles se réunissaient dans le réfectoire des frères prêcheurs.

15 Ces sociétés exprimaient par des pétitions l'opinion des dames, indépendamment de celle des hommes.

16 Les Dames veulent être intégrées à la cérémonie de la fête de la Fédération du 14 juillet 1790 et prêter le serment civique comme le montre une délibération publiée issue de la séance du 7 juillet 1790 des Dames citoyennes du district de Saint-Martin. Ayant affirmé que les principes de la Déclaration des droits de l'homme et du citoyen s'adressaient aux deux sexes, elles veulent jurer comme les hommes de défendre la Constitution. Les Dames estiment que les femmes sont régénérées par la Révolution et qu'elles ne doivent plus être considérées comme les créatures frivoles de l'Ancien Régime; elles ont été libérées du despotisme et sont prêtes à repousser avec un " mâle courage ", les ennemis du bien public qui tenteraient de les « replonger dans les fers $»^{6}$.

17 L'argumentation des Dames citoyennes comme celle des Dames hérö̈nes est largement fondée sur le passé marseillais. La référence aux dames de Marseille, héroönes du siège de 1524, qui avaient rejoint les hommes pour défendre la ville contre les troupes du connétable de Bourbon, est très forte dans leurs propos ${ }^{7}$. Cet exemple historique, qui fait partie de la mémoire collective de la ville ${ }^{8}$, semble avoir beaucoup aidé les patriotes marseillaises qui n'ont pas besoin de se référer aux exemples lointains des Romaines et des Gauloises pour revendiquer une place aux côtés des hommes dans la défense de la patrie, de la Révolution. La notion de vertu héroïque, qui permet aux femmes de surmonter leur faiblesse naturelle, était déjà présente dans les éloges des Dames de Marseille des concours poétiques du XVIII ${ }^{\mathrm{e}}$ siècle. On voit comment le danger et le conflit permettent aux Marseillaises de s'exprimer par le biais de cette identification à leurs glorieux ancêtres. Le nom des Dames héroïnes en est directement inspiré. Les Dames citoyennes veulent aussi participer à la défense de la cité, disant «préférer la mort à l'humiliation des chaînes ». Elles proposent, à l'exemple des héroïnes passées, de se battre avec un «mâle courage ». Il s'agit à cette époque d'une revendication de défense de la Nation plutôt symbolique, mais les Dames forment elles aussi leur milice. Les responsables des Dames citoyennes étaient les épouses des officiers de la garde nationale de Marseille. Les Dames héroïnes se constituent en bataillons. Madame Lieutaud, la femme du commandant de la garde nationale, devient leur générale, de même major et capitaine sont des femmes d'officiers de la garde nationale. Madame Lieutaud et Madame Martin sont les épouses de négociants bien connus ayant exercé des fonctions municipales, ce qui a facilité les choses pour que les Dames citoyennes obtiennent 
l'approbation de la municipalité. En janvier 1790, Étienne Martin, candidat du parti populaire aux municipales, l'avait emporté sur le parti aristocrate.

Le 13 juillet 1790, une déclaration avait été envoyée à l'Assemblée nationale pour approuver ses « immortels décrets » et la "sainte Constitution ». À partir de la séance du 7 juillet 1790, "leurs cœurs enflammés par le patriotisme», les Dames affirmaient vouloir prêter le serment civique et marcher sous le drapeau de la liberté. La délibération des Dames citoyennes du 7 juillet mettait l'accent sur le fait que les « heureuses destinées » promises par la Constitution concernaient l'un et l'autre sexe.

C'est, en effet, le patriotisme qui autorise les femmes à s'exprimer publiquement ; elles le font au niveau local et national. Les deux sociétés féminines agissent ensemble pour obtenir de pouvoir prêter serment. À la veille du 14 juillet, les Dames citoyennes diffusent un « avis pressant » invitant chacun des 25 districts de « l'armée féminine » à dresser à l'occasion de la fête une table de 200 couverts où les nécessiteux seraient servis par les dames, assumant ainsi publiquement le rôle traditionnellement charitable attribué aux femmes.

20 Les autorités municipales qui avaient accepté que les dames tiennent des réunions politiques acceptent aussi qu'elles participent à la fête et prêtent le serment civique. La proclamation municipale reprend les propos des Dames, qui se comparent explicitement aux héroïnes du XVI ${ }^{\mathrm{e}}$ siècle. Néanmoins, les Marseillaises ne prêtent pas le serment le 14 juillet, comme elles l'auraient souhaité, "avec leurs époux-citoyens, leurs enfants et leurs frères ${ }^{9}$, mais le 15 séparément et après les hommes ${ }^{10}$.

21 Le 15 juillet 1790, elles se rassemblent d'abord dans leurs districts dont chacun avait son étendard, toutes vêtues de blanc avec une ceinture tricolore et une cocarde tricolore au bras gauche, tenant à la main une branche de laurier. La femme du maire et celle du commandant de la garde nationale en tête, elles gagnent la maison commune où les attendent les autorités municipales. Elles sont ensuite escortées d'un détachement de la garde nationale et d'un bataillon de la marine. À l'autel de la patrie, le maire prononce un discours où il évoque les héroönes du siège, mais pour indiquer que le temps n'est plus de ce type d'action, que la liberté est conquise et qu'il ne s'agit que de la conserver. Martin, politiquement modéré, met l'accent sur la tranquillité publique que les Dames doivent concourir à maintenir autant que la Constitution. Il préfère faire appel à leurs talents de persuasion qu'à leur courage. Les Dames dont nous n'avons pas la réponse prêtent le serment ; Te Deum, salve d'artillerie, danses et farandoles s'ensuivent. D'après le journaliste Ferréol Beaugeard, cette fête vraiment nouvelle avait attiré un nombre prodigieux de spectateurs ${ }^{11}$.

22 Si les Dames hérö̈nes n'apparaissaient que pour la fête de la Fédération, le nombre des Dames citoyennes a pratiquement doublé en 1790. Les dirigeantes de ces clubs sont des bourgeoises, mais les sections 7 et 11 étaient en plein cœur de la vieille ville et comportaient des quartiers pauvres. Ces deux sections adjacentes et proches des quartiers populaires du port de Marseille ont une composition sociale mixte ${ }^{12}$.

23 Au-delà de la fête, les Dames citoyennes participent publiquement aux conflits politiques marseillais. Les Dames citoyennes de la section Saint-Martin $n^{\circ} 7$ publient une dénonciation d'André, commissaire du roi envoyé à Marseille à l'automne 1789, qui avait lui-même dénoncé les autorités municipales et le commandant de la garde nationale Lieutaud. Dans ce texte, elles montrent comment c'est le péril qui les autorise à prendre publiquement la parole. Elles parlent, en ces circonstances, de «l'obligation» de faire entendre leur voix, en dérobant quelques instants aux occupations de leurs ménages ${ }^{13}$. 
Le texte est argumenté, très renseigné sur la situation marseillaise, et violent. Les Dames font référence aux Lacédémoniennes capables de sacrifier leurs enfants pour la patrie et s'affirment elles-mêmes capables de mourir pour sauver la Constitution. La référence aux Dames du siège est à nouveau présente pour convaincre que le patriotisme peut l'emporter sur la faiblesse que l'on reproche au sexe féminin.

Les femmes sont bien accueillies au club des Jacobins Le 7 novembre 1790, Marie Martin y prononce un éloge des Dames citoyennes. Son discours est publié $^{14}$. Marie Martin souligne le patriotisme des Dames, l'énergie qu'elles apportent à soutenir la Révolution. Elle met l'accent sur les liens entre citoyenneté, patriotisme, sentiments et devoirs. Bien sûr, le rôle d'éducatrice des femmes est mis en valeur pour enseigner le patriotisme et les devoirs inhérents :

« Heureuses sont celles qui, donnant des enfants à la patrie, et serrant dans leurs bras ces tendres fruits d'un amour conjugal, feront sucer avec le lait, ces grands principes d'égalité, cet amour ardent pour la patrie, pour la liberté, et cet attachement inviolable à la Constitution. ${ }^{15}$

Les patriotes se veulent citoyennes parce qu'elles participent à chaque stade à la création de l'homme nouveau, du futur citoyen. Le rôle traditionnel d'épouse et de mère est transformé par la Révolution. Le sein de la mère devient politique. De ce fait, les femmes veulent pouvoir librement choisir des maris patriotes. Marie Martin, ellemême encore célibataire, affirme, au nom de toutes ses compagnes qu'aucun homme ne doit aspirer à s'unir à elles s'il n'est un des fermes soutiens de la liberté naissante ${ }^{16}$. Le concept politique de la citoyenne épouse et mère et les devoirs qui y sont attachés politisent le mariage, la maternité, l'espace privé17.

L'analyse des adresses patriotiques que le club envoie à l'Assemblée nationale montre l'importance qu'il accorde dans l'évolution politique aux transformations du mariage et des relations familiales. D'ailleurs, ces femmes pratiquent souvent la politique en famille, mères, filles, belles-filles aux Dames citoyennes, pères, fils et gendres aux Jacobins. Cette participation politique de familles entières se retrouvera aussi dans le mouvement sectionnaire marseillais. Mais les Dames s'expriment également en dehors de l'espace maternel et familial comme le montre leur soutien à la Constitution, leur dénonciation d'André.

La révolution marseillaise porte ensuite au pouvoir des Jacobins plus radicaux que Lieutaud et Martin, époux des principales responsables des Dames citoyennes. Favorable à la monarchie constitutionnelle, Étienne Martin est élu à la Législative en août 1791 et remplacé par un maire jacobin républicain, Mouraille. Par contre, Lieutaud et ses partisans ont été évincés du pouvoir en octobre 1790 ; il finit par être considéré comme contre-révolutionnaire. À partir de 1791, il n'y a plus de trace des Dames citoyennes, mais les femmes ne disparaissent pas de l'espace public, elles participent aux séances du club des Jacobins et aux assemblées des sections.

Les papiers d'une autre société populaire féminine ont été retrouvés, il s'agit de celle de Castellane, dans les Basses-Alpes ${ }^{18}$. Le club féminin est fondé, le 3 juin 1792, avec l'aide d'hommes patriotes, après le passage de missionnaires patriotes marseillais. C'est d'ailleurs le club masculin qui, après avoir délibéré s'il invitait les Dames patriotes à venir assister aux séances, avait résolu de les inciter à former elles-mêmes une Société des amies de la Constitution. Les Dames s'affilient ensuite à la société masculine et en sont dépendantes ; elles avaient demandé deux commissaires masculins qui puissent suivre leurs travaux et les instruire. Néanmoins elles ne craignent pas d'affirmer des 
idées d'émancipation comme dans ce discours de la présidente s'adressant aux clubistes masculins :

« Frères et amis, c'est avec une grande satisfaction que nous venons vous exprimer nos sentiments qui ne le cèdent en rien aux vôtres pour le patriotisme, jusqu'à ce jour vous nous avez regardé comme vos idoles, mais lors même que vous sembliez ne recevoir de lois que de nous, nous étions vos esclaves et vous ne nous regardiez que comme un joujou qui servait à vous amuser et vous distraire pour les conseils et les affaires. »

Mais la suite du discours montre que ces femmes patriotes se résignent à la différenciation des rôles politiques :

«[...] condamnées par les loix de notre sexe à nous occuper dans l'intérieur, que des affaires qui ont rapport à nos ménages, nous pouvons être utiles et maintenir la Constitution, non seulement en inspirant du courage mais en prêchant la paix et l'union. »

La présidente, Madame Blanc, née de Roffi, écrit le 8 juin au président de la Société des amis de la Constitution de Digne une lettre qui est lue à la séance du 10 juin de ce club, centre des sociétés du département, leur "mère commune " comme le dit la présidente ${ }^{19}$. Cette lettre est jugée digne d'être imprimée, elle doit avoir été expédiée aux autres sociétés du département puisque Patrice Alphand en a retrouvé la trace à Riez.

Comme dans le discours précédent, la présidente met l'accent sur l'oubli des femmes pendant l'Ancien Régime et les " préjugés indécents » qu'on attachait à leur sexe. Avec la Révolution, « un nouveau jour va luire pour le sexe». Les législateurs ayant confié aux mères de famille le soin d'instruire leurs enfants des lois de la «sublime constitution ", elles doivent elles-mêmes s'en instruire et ne peuvent le faire qu'en "puisant les vrais principes" dans les sociétés patriotiques. Grâce aux soins de la philosophie et d'une nouvelle éducation, les femmes seront aptes à tenir le rôle qui leur a été attribué. L'argumentation de la présidente se fonde donc essentiellement sur le rôle de mère et d'éducatrice reconnu aux femmes pour justifier leur participation à la sociabilité politique. Elle réclame l'aide de leurs « frères et amis » pour les instruire et les encourager, ce qu'elle semble obtenir sans problème.

Le 17 juin 1792, les Amies de la Constitution furent invitées à se joindre à leurs frères pour assister à leurs séances. Les Dames acceptèrent, mais leur société «conserverait son caractère distinct indépendant et aurait une présidente qui figurerait avec le président et les soeurs auraient voix comme membre de la Société des frères et amis $»^{20}$. Lors de la séance, un moment après le président, arrive « Madame la Présidente » suivie de toute la société à qui on attribue le côté droit et le fauteuil. Néanmoins, la société siégeant avec les hommes perd de sa spécificité et il n'y a plus de mention des Dames jusqu'au 9 avril 1793. On invite alors «la citoyenne présidente de notre aimable sexe à tenir leur assemblée ". Il continue donc à y avoir deux sociétés qui se rejoignent pour de nombreuses séances. La situation de la société de Castellane confirme le fait, qu'en province, il ne semble guère y avoir de tensions entre les sociétés masculines et féminines qui se composent d'ailleurs des membres des mêmes familles.

Dans les clubs "mixtes", les femmes s'expriment relativement peu ; dans de nombreuses sociétés, elles doivent se contenter d'assister aux séances. À Aix-enProvence, elles ne peuvent faire ni motion, ni discours, ce qui n'est pas le cas à Marseille. L'interdiction des clubs de femmes ne change rien pour les Marseillaises qui sont déjà passées de clubs féminins à une participation aux Jacobins et aux sections. 
Le Journal des départements méridionaux ${ }^{21}$ signale la présence régulière de femmes au club marseillais de la rue Thubaneau. Elles y occupent les tribunes. Elles participent aux dons patriotiques, aux fêtes, fabriquent des vêtements pour les soldats, mais elles prononcent aussi des discours politiques, s'exprimant dans la sphère publique et se voulant les porte-parole des autres citoyennes. D'après le Journal, leurs discours sont très applaudis. Le 27 février 1793, la citoyenne Chabert présente un discours au cours de la séance de la société. Ce dernier est ensuite publié dans le Journal des départements méridionaux ${ }^{22}$. La citoyenne Chabert s'exprime dans le contexte de la patrie en danger. Après avoir rappelé le passé révolutionnaire de Marseille depuis 1789, elle encourage à la mobilisation patriotique :

«[...] Nous espérons que Neptune vous protégera, et que vous reviendrez couverts de lauriers. Nos mains sont prêtes à vous en ceindre, comme nous sommes prêtes à endosser vos uniformes pour venger les héros, qui auront fini glorieusement leur vie pour la défense de la République. Qu'en dites-vous, citoyennes qui m'entendez? Ne sommes-nous pas toutes du même sentiment ? La même ardeur ne nous animet-elle pas? Je vois d'avance vos cœurs hérö̈ques s'enflammer ; je vous vois prêtes à marcher au premier signal, pour concourir au bonheur commun. Oui, citoyens, nous jurons toutes de remplacer nos frères d'armes qui auront été enlevés par les Parques cruelles! Nous jurons toutes de suivre les traces des anciennes Romaines, de ne prendre pour époux que de vrais sans-culottes, et de ne reconnaître d'autre maitre que Dieu, et nul autre souverain sur la terre que le Peuple. »

Le 21 avril 1792, Madame Macaire présente l'adresse d'un groupe de citoyennes demandant à être armées pour la défense de la liberté, avec toujours la référence au siège de 1524. Les revendications de porter les armes sont fréquentes à l'époque de la patrie en danger. Le missionnaire patriote Montbrion, dans un compte rendu de sa mission, en 1792, met en valeur les citoyennes d'Éguilles qui ont formé une compagnie, disant « dans le temps que nos maris s'occuperont à labourer la terre, il faut que nous soyons armées de leurs fusils pour veiller au salut de la chose publique ». Les Monnaidières arlésiennes, armées de sabres, accompagnent les hommes pour leurs expéditions punitives ${ }^{23}$.

Néanmoins, dans leurs interventions, les femmes mettent plus souvent en avant leurs rôles de filles, d'épouses, de mères qui leur permettent de jouer un rôle moteur dans la communauté que leur volonté de créer des légions d'Amazones.

Les femmes dans les sections

37 L'engagement des femmes pendant la crise de 1793 est encore assez mal connu. Cependant divers indices permettent de déceler leur présence tant dans les sections fédéralistes qu'au sein du mouvement de résistance au fédéralisme. Les archives judiciaires prouvent que des femmes ont participé au mouvement sectionnaire du printemps et de l'été 1793, mais les registres des sections ont été détruits en grande partie.

38 À Carpentras, des dépositions contre la citoyenne Amiel montrent qu'elle assistait aux séances des sections et qu'elle y prenait la parole ; un patriote arrêté à cette époque l'accuse d'avoir demandé qu'il soit guillotiné24. C'est, en effet, souvent par les dénonciations portées par ces femmes contre les Jacobins au moment où les sections dominaient dans leurs communes respectives que l'on peut saisir leur trace, soit directement quand l'accusation a été conservée, soit par les contre-dénonciations devant les comités de surveillance qu'elles subissent en l'an II. Plusieurs exemples peuvent être trouvés à Eguilles dont le comité de surveillance avait pris soin d'aller 
recopier à Aix-en-Provence les accusations portées pendant la période fédéraliste ${ }^{25}$. De nombreuses femmes avaient alors été dénonciatrices, le plus souvent de contributions forcées ${ }^{26}$.

À Marseille, où le mouvement sectionnaire prend le pouvoir au printemps 1793, les femmes occupent les tribunes des assemblées de leurs sections respectives et sont présentes par leurs propos et leurs acclamations dans les assemblées sectionnaires, sans pour autant participer aux délibérations.

Les recherches menées par Jacques Guilhaumou témoignent que des citoyennes ont demandé à participer aux assemblées de section de leur quartier, en particulier au moment de la campagne anti-clubiste ${ }^{27}$. Ces demandes provoquent d'ailleurs une certaine résistance masculine, ainsi à la section 16 le 20 mai 1793 :

"Le président a lu une adresse des citoyennes de la section qui demandent à l'assemblée d'assister à ses séances en se plaçant dans les tribunes, sur quoi la discussion n'ayant pu amener aucune décision, il a été délibéré de nommer huit commissaires qui seront chargés par l 'assemblée d'examiner les inconvénients et les avantages qui peuvent résulter de la présence des femmes aux délibérations de la section, pour en faire un rapport et donner leur avis. ${ }^{28}$

41 Lorsque les sections se décident à accepter les citoyennes, elles exigent qu'elles soient séparées des hommes puisque lorsque les tribunes leur sont accessibles, elles deviennent interdites aux hommes. Ces femmes sectionnaires appartiennent à des familles impliquées dans le mouvement fédéraliste, elles sont, le plus souvent, issues du monde de la boutique et de l'artisanat, mais on y trouve aussi des domestiques. Quelques documents témoignent de leur activité : le 22 mai, une députation de femmes de marins est reçue à la section 4 , le 26 mai une adresse de citoyennes républicaines y est lue et enfin une pétition de citoyennes le 29 mai $^{29}$.

42 Certains documents, analysés par Jacques Guilhaumou dans Marseille républicaine ${ }^{30}$, montrent l'influence du sexe féminin dans la mobilisation sectionnaire. La rhétorique de «la sensibilité émue » occupe alors une place grandissante dans le discours, avec l'énonciation de la volonté de concourir au bonheur commun ${ }^{31}$. Cette rhétorique des passions est centrée sur le thème de l'héroïsme.

43 Le 7 août 1793, une femme (anonyme) rappelle aux citoyens de la section 5 leur devoir patriotique face à la menace des armées de la Convention ${ }^{32}$. Elle commence par justifier l'influence des femmes qui, de tout temps "polissent les mours, élèvent l'âme, ornent l'esprit »; en période de crise, elles peuvent sortir de ce rôle civilisateur en « ajoutant l'exemple de l'héroïsme ".

44 «C'est surtout dans une république naissante qu'il faut électriser les âmes. Un tel état a besoin pour se raffermir du développement de toutes les facultés humaines et du ressort actif des passions ennoblies par l'amour de la Patrie et de l'Humanité [...]

Mais à qui mieux qu'à nous appartient-il d'exciter l'ardeur des braves descendants des Phocéens : ignore-t-on l'ascendant vainqueur d'un sexe faible en apparence?

Élevés par nos soins, nos enfants, n'en doutez pas, apprendront de bonne heure que leur titre le plus sacré est celui de citoyen. Ils sauront que leur premier devoir est de mourir pour la Patrie [...]

Que l'amour, cette passion efféminée dans un gouvernement despotique, soit dans notre république le premier mobile de toutes les vertus. N'allumons son flambeau que pour conduire nos époux à la victoire. Lui seul peut donner cette mâle énergie, première vertu de l'âme pour former le héros [...]». Elle argumente ensuite sur le 
thème du combattant rempart de son foyer : «Si vous reculez, nous périssons tous par le glaive des assassins [...] Ah ! Frémissez du spectacle d'horreur qui s'offrirait à vos yeux si, contre notre attente, vous ne vous empressiez de rejoindre vos drapeaux. Sachez que quelques uns de vous n'échapperaient pas à la mort que sur les corps sanglants d'une mère ou d'une épouse expirante [...] » Après avoir dressé un sombre tableau des meurtres et viols qui suivraient la reprise de Marseille par les armées de la Convention, l'oratrice fait briller l'espoir d'une victoire récompensée par le triomphe que feront aux combattants leurs filles et leurs femmes. Elle termine en demandant que sa motion soit convertie en pétition et envoyée à l'adhésion des autres sections pour « qu'au retour de nos braves guerriers, ils soient couronnés par nos mains sur l'autel de la Patrie $»^{33}$.

45 Cette pétition qui, finalement, ne propose pas de sortir les femmes de leur rôle traditionnel est fortement applaudie, la section faisant, elle aussi, référence au courage des Marseillaises lors du siège de 1524.

Le registre de la section 4 du 9 août 1793 cite le discours de Thérèse Clappier, âgée de 16 ans, fille d'un parfumeur, arrêtée ainsi que ses parents pour fédéralisme. Elle est accusée de se servir de ses charmes pour "pervertir les esprits et corrompre les mœurs ». Elle se défend d'avoir prononcé le discours contre-révolutionnaire qui lui est reproché et dit qu'il avait été écrit par un homme : «C'est le citoyen Maisse qui me l'avait fait pour me faire passer pour une héroïne, je ne l'ai pas prononcé ${ }^{34}$. La fille de Maisse, Sabine, est une amie de Thérèse, avec qui elle se rend aux assemblées de la section 4.

Les analyses menées par Jacques Guilhaumou mettent l'accent sur ces jeunes républicaines, formées à l'école de la souveraineté du peuple, instruites des droits de l'homme qui symbolisent par leur présence et leurs discours, la dimension " héroïque " de tout moment d'enthousiasme. Elles contribuent ainsi à l'élargissement de l'action politique aux spectateurs de l'événement ${ }^{35}$.

À de rares et précieuses exceptions près, la parole des femmes est surtout accessible à travers des documents issus de la répression, qu'elle s'exerce d'un côté ou de l'autre. Ainsi, c'est à l'occasion de dépositions devant les comités de surveillance ${ }^{36}$ ou les tribunaux qu'on s'aperçoit que des femmes qui sont dénoncées pour leurs propos ont pris la parole en public. Cet état de fait provoque un certain déséquilibre en faveur des femmes opposées à la Révolution, ce qui a contribué à la constitution de l'image d'un engagement politique féminin majoritairement contre-révolutionnaire.

Dénonciations et contre-dénonciations témoignent de l'importance de la parole féminine dans les pratiques politiques villageoises et populaires urbaines ${ }^{37}$ : elles sont dénoncées pour avoir fait circuler de fausses nouvelles, pour avoir critiqué les mesures prises par le camp au pouvoir, pour avoir menacé les adversaires. Ces propos ont souvent pour but de convaincre les autres habitants et donc de recruter pour leur propre camp. Des femmes du peuple ne craignent pas d'affirmer violemment, en public, leurs idées politiques traitant les députés de voleurs et l'Assemblée de "chambre des couyons $\aleph^{38}$. Des femmes s'affirment publiquement pour la défense de la religion, se mettant davantage en visibilité que les hommes quand il s'agit de lutter pour des convictions religieuses ${ }^{39}$.

De quelque bord qu'ils soient, les hommes expriment souvent leur crainte du pouvoir des mots des femmes. La "mauvaise langue » des femmes est fréquemment dénoncée par les autorités en place. C'est dans les lieux de sociabilité traditionnels que les femmes se servent de leur parole pour mener une action de propagande politique, au 
service de l'un ou l'autre camp. Cette parole s'exprime en dehors de cadres institutionnels mais dans l'espace public au sein duquel les femmes contribuent à la constitution de l'opinion, dès l'Ancien Régime.

51 Les femmes semblent s'exprimer de façon privilégiée en situation de crise, ou peut-être leur parole est-elle mieux entendue quand cela va mal. Le danger qui les menace, elles et leurs enfants, leur donne le droit de se faire entendre. La notion de défense est très forte dans les textes qui nous sont parvenus, qu'il s'agisse de défendre la patrie, la République, la religion, l'ordre... Les demandes pour porter les armes ont leur justification dans cette volonté de défense. Les patriotes utilisent la rhétorique révolutionnaire pour exprimer leurs opinions et leurs rôles d'épouses et de mères pour participer en tant que citoyennes à l'espace public. Même lorsqu'elles prennent la parole dans des clubs mixtes, les femmes se pensent comme telles, différentes des hommes et se voulant représentatives des autres femmes, bien qu'elles rejoignent les orientations politiques et les combats des hommes.

De façon générale, mais en particulier dans le mouvement fédéraliste, les femmes utilisent le registre de l'apitoiement (les hommes doivent combattre pour défendre femmes et enfants), celui de la sensibilité, de la compassion, de l'humanité ; elles se situent dans une rhétorique du sentiment.

53 Les femmes s'expriment dans tous les camps politiques et, d'après les documents en notre possession, souvent issus de la répression, il est bon de le rappeler, leurs positions apparaissent plus souvent radicales que conciliatrices. Elles poussent les hommes à l'action et jouent davantage un rôle de boute-feux, comme l'ont montré les travaux de Dominique Godineau sur les Parisiennes, que de médiatrices.

Une table ronde, organisée à Aix-en-Provence à l'occasion des journées «Femmes et politique $\aleph^{40}$, animée par Dominique Godineau, Christine Peyrard et Michel Vovelle, avait permis de comparer les attitudes politiques des Provençales à celles des Parisiennes mais aussi à celles qui ont été étudiées dans d'autres régions comme l'Ouest. Le débat avait montré qu'en dépit de spécificités locales, les mêmes stratégies ont été empruntées par les femmes pour tenter de faire entendre leurs voix dans l'espace public ${ }^{41}$.

Néanmoins, dans l'état actuel de recherches qui se poursuivent, il semble que les Provençales ne se mobilisent pas pour le droit de vote des femmes, ni pour leur accès aux fonctions publiques. Par leurs paroles, comme par leurs actes, elles "se contentent » de participer à la vie politique faite d'antagonismes de la région.

\section{NOTES}

1.Voir Martine LAPIED, «Conflictualité urbaine et mise en visibilité des femmes dans l'espace public de l'Ancien Régime à la Révolution en Provence et dans le Comtat Venaissin ", Provence Historique ${ }^{\circ} 202,2001$.

2.Voir dans Paule-Marie DUHET, Cahiers de doléances des femmes et autres textes, Des Femmes, 1981. 
3.Voir l'enquête sur les sociétés populaires de Jean BoutIER et Philippe BOUTRY, AHRF., $n^{\circ} 266$ (sept.- oct. 1986).

4.D'après Suzanne DesAN, « Constitutionnal Amazons. Jacobin Women's Clubs in the French Revolution », dans Re-creating Authority in Revolutionary France, Rutgers

University Press, New Jersey, 1992.

5.Voir dans Paule-Marie DuHET, Cahiers de doléances des femmes et autres textes, op. cit. 6.Délibération des Dames citoyennes du district Saint-Martin du 7 juillet 1790.

7.Cf Laura Emerson TAlamante, Les Marseillaises: Women and Political Change During the French Revolution (1789-1794), Doctorat de l'Université de Californie, sous la direction de Lynn HuNT, 2003.

8.Voir Hélène ÉCHINARD, « Les Dames du siège de Marseille (1524) », dans Le Panthéon des femmes. Figures et représentations des Héroïnes, sous la direction de Geneviève DERMENJIAN, Jacques Guilhaumou, et Martine LAPIED, Paris, Publisud, 2004.

9.Délibération des Dames citoyennes du district Saint-Martin du 7 juillet 1790.

10.Voir Yvonne KNIBIEHLER, «Femmes de Provence en révolution », dans Les femmes et la Révolution française, sous la direction de Marie-France BRIVE, Toulouse, Presses Universitaires du Mirail, 1989, t.1, p. 149-155.

11.Journal de Provence, t. 28, p. 279.

12.Voir William SсотT, Terror and Repression in Revolutionary Marseille, Cambridge, New York, Cambridge University Press, 1985.

13.Dénonciation du sieur André par les citoyennes de la section Saint-Martin.

14.Le Patriotisme des Dames citoyennes; discours prononcé à la tribune de l'Assemblée Patriotique par Mademoiselle Marie Martin le 7ème novembre de l'an II de la Liberté, Marseille, 1790.

15.Ibid.

16.Ibid.

17.Voir Martine LAPIED, « Les femmes entre espace privé et espace public pendant la Révolution française ", dans Georges Duby, regards croisés sur l'œuvre. Femmes et féodalité, sous la direction d'Annie Bleton-Ruget, Marcel Pacaut et Michel Rubellin, Lyon, Presses Universitaires de Lyon, 2000.

18.Les papiers des sociétés populaires des Basses-Alpes ont été étudiés par Patrice ALPHAND dans un mémoire de maîtrise de l'Université de Provence, dirigé par Michel VovelLE en 1983, travail dont il a tiré le chapitre « Les sociétés populaires » dans « La Révolution dans les Basses Alpes », bulletin n 307 des Annales de Haute-Provence, 1989, p. 288 à 350.

19.AD Alpes de Haute Provence, L 852.

20.AD Alpes de Haute Provence, L 301.

21.Journal des départements méridionaux édité par Joseph GIRAUD, Alexandre RICORD fils et Pierre Micoulin, 184 numéros du 6 mars 1792 au 7 mai 1793, Bibliothèque de Marseille, 1716-1717.

22.Ibid., 156, 2 mars 1793.

23.Voir Martine LAPIED, «Les Provençales actrices de la Révolution ? L'exemple des Arlésiennes ", dans Pour la Révolution française, recueil d'études en hommage à Claude MAZAURIC réunies par Christine LE Bozec et Éric WAUTERS, Publications de l'Université de Rouen, 1998.

24.AD Vaucluse, $6 \mathrm{~L} 34$.

25.AD Bouches-du-Rhône, L 1768. 
26.Voir Martine LAPIED, « Une communauté provençale pendant la Révolution française : les antagonismes politiques à Eguilles », dans « De Provence et d'ailleurs », mélanges offerts à Noël Coulet réunis par Jean-Paul BOYER et François-Xavier EMMANUELLI, Provence Historique n 195-196, 1999.

27.Voir Jacques GuILHAumou, « Conduites politiques de Marseillaises pendant la Révolution française ", dans Femmes et politique en Provence XVIII ${ }^{e} X^{e}$ siècles, dir. Martine LAPIED et Éliane RICHARD, Provence Historique n 186, 1996.

28.AD Bouches-du-Rhône L 1944.

29.Voir Jacques GuILHAUMOU, « Conduites politiques de Marseillaises pendant la Révolution française ", dans Femmes et politique en Provence XVIII ${ }^{e}$ XX $X^{e}$ siècles, op. cit. 30.Jacques Guilhaumou, Marseille républicaine (1791-1793), Paris, Presses de la Fondation nationale des sciences politiques, 1992.

31.Voir Jacques GuILHAUMOU, « Une identité politico-linguistique éphémère ; le républicanisme provençal (1790-1793)», CAER XVIII, Publications de l'Université de Provence, 1995.

32.Extrait du registre des délibérations de la section 5, séance du 7 août 1793 (AD Bouches-du-Rhône L 1984).

33.Le texte entier de la motion est cité dans Jacques GuILHAUMou, Marseille républicaine (1791-1793), op. cit., p. 245-248.

34.AD Bouches-du-Rhône L 3119.

35.Jacques GuilHaUmou, « Conduites politiques de Marseillaises pendant la Révolution française ", op. cit.

36.Voir Jacques GuILHAUMou et Martine LAPIED, « Les femmes dans les archives des comités de surveillance ", dans Femmes entre ombre et lumière. Recherches sur la visibilité sociale (XVI ${ }^{e}-X^{e}$ siècles), sous la direction de Geneviève DERMENJIAN, Jacques GUILHAUMOU, Martine LAPIED, Publisud, 2000.

37.Voir Martine LAPIED, « La place des femmes dans la sociabilité et la vie politique locale en Provence et dans le Comtat Venaissin sous la Révolution française ", dans Femmes et politique en Provence, op. cit.

38. Comité de surveillance de Grasse, AD des Alpes Maritimes, L 921-923.

39.Voir Martine LAPIED, « Les Comtadines et la Révolution française : une défense prioritaire de l'identité religieuse? ", actes du colloque Religion et identité, sous la direction de Gabriel AUDISIO, UMR Telemme, Publications de l'Université de Provence, 1998.

40.Journées organisées à l'occasion de la célébration du cinquantenaire du premier vote des femmes en France par le groupe de recherches Femmes Méditerranée de la MMSH, l'UMR Telemme (Université de Provence - CNRS), l'association Les femmes et la ville.

41.Cf « Femmes et politique en Provence (XVIII ${ }^{\mathrm{e}}-\mathrm{XX}^{\mathrm{e}}$ siècles) », numéro spécial de Provence historique, fasc. 186, 1996. 


\section{RÉSUMÉS}

Il existe relativement peu de traces de la parole publique des femmes « ordinaires », néanmoins des recherches approfondies dans les fonds d'archives montrent qu'elle a existé. Cette parole devient plus facilement visible en situation de crise, de conflictualité, dans les périodes d'affaiblissement des normes qui voudraient cantonner les femmes à l'espace privé. À partir d'un corpus de sources essentiellement issues des papiers des sociétés populaires, comités de surveillance et tribunaux provençaux, l'étude tente de faire apparaître cette parole publique des femmes et de montrer les voies que les Provençales empruntent pour être entendues dans une région où les antagonismes politiques sont prononcés.

The Public Voice of Women and Conflict during the Revolution in the Southeast of France. There are relatively few traces of the public voice of «ordinary» women. Yet in-depth archival research shows that they existed. This voice is more easily recognizable during times of crisis, of conflict, in periods of weakening of norms that restrict women to the private space. Using a collection of sources consisting essentially of documents from societe populaires, comites de surveillance, and courts in Provence, this study aims to document the public voice of women, and to show the ways by which Provencal women made themselves heard in a region where political hostilities were pronounced.

INDEX

Mots-clés : jacobins, sociétés populaires, doléances, clubs féminins, sections

\section{AUTEUR}

\section{MARTINE LAPIED}

Professeur à l'Université de Provence, MMSH, 5 rue du Château de l'Horloge, BP 647, 13094 Aix-en-Provence cedex 2, martine.lapied@wanadoo.fr 